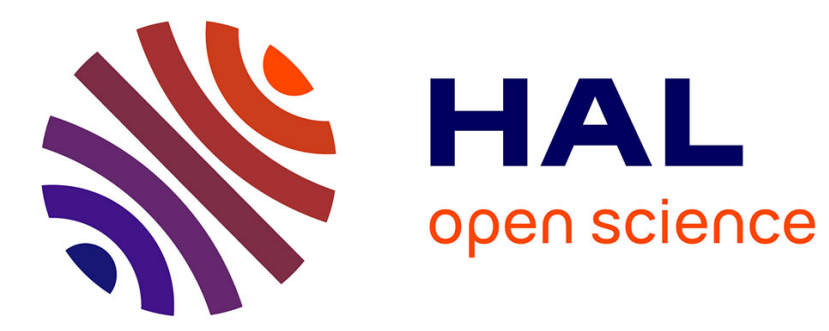

\title{
Time-Domain Simulation of Sampled Weakly Nonlinear Systems Using Analytical Integration and Orthogonal Polynomial Series
}

\author{
Ewout Martens, Georges Gielen
}

\section{- To cite this version:}

Ewout Martens, Georges Gielen. Time-Domain Simulation of Sampled Weakly Nonlinear Systems Using Analytical Integration and Orthogonal Polynomial Series. DATE'05, Mar 2005, Munich, Germany. pp.120-125. hal-00181504

\author{
HAL Id: hal-00181504 \\ https://hal.science/hal-00181504
}

Submitted on 24 Oct 2007

HAL is a multi-disciplinary open access archive for the deposit and dissemination of scientific research documents, whether they are published or not. The documents may come from teaching and research institutions in France or abroad, or from public or private research centers.
L'archive ouverte pluridisciplinaire HAL, est destinée au dépôt et à la diffusion de documents scientifiques de niveau recherche, publiés ou non, émanant des établissements d'enseignement et de recherche français ou étrangers, des laboratoires publics ou privés. 


\title{
Time-Domain Simulation of Sampled Weakly Nonlinear Systems Using Analytical Integration and Orthogonal Polynomial Series
}

\author{
Ewout Martens* and Georges Gielen \\ Katholieke Universiteit Leuven, Department of Electrical Engineering, ESAT-MICAS \\ Kasteelpark Arenberg 10, B-3001 Leuven, Belgium \\ \{emartens|gielen\}@esat.kuleuven.ac.be
}

\begin{abstract}
This paper presents a novel method for simulation of sampled systems with weakly nonlinear behavior. These systems can be characterized by adding weakly nonlinear terms to the linear state-space equations of the system resulting in an extended state-space model. Perturbation theory is used to split these equations in an ideal linear behavior and a non-ideal small perturbation. The linear equations are solved analytically which reduces simulation time compared to numerical evaluation. The solution of the perturbation equations is approximated by orthogonal polynomials.

This methodology not only reduces simulation time compared to traditional numerical simulations, but also deals naturally with clock jitter and the discontinuous behavior of sampled systems. An implementation of the methodology has been used to analyze systems including switched filters and continuous-time $\Delta \Sigma$ modulators.
\end{abstract}

\section{Introduction}

Modern integrated circuits frequently contain sampled subsystems. In mixed analog-digital systems, conversions between the analog and the digital domain are key operations. Further, a part of the processing algorithm can be implemented in the discrete domain, using for example switched-capacitor circuits.

Methodologies commonly used for systematic analysis and synthesis of analog circuits require timeefficient simulations to determine and optimize the performance [3]. Using these methods for sampled systems implies executing simulations which take into account major non-idealities like clock jitter, charge injection and weakly nonlinear behavior [5]. Furthermore, signals encountered in switched circuits have

\footnotetext{
${ }^{*}$ Research Assistant of The Fund for Scientific Research - Flanders (Belgium)
}

inherently a discontinuous time behavior making it difficult to use simulators often used by analog designers like SPICE.

Simulation of sampled systems can be performed in time or frequency domain [9]. The goal of this work is to develop more efficient time-domain simulation of weakly nonlinear sampled systems which can be used as part of simulations of larger mixed-signal systems with a clock-based computational model. Applications are, for example, time-domain analysis of sensor interfaces with switched-capacitor filters and $\Delta \Sigma$ converters.

The approach presented in this paper is based on splitting up the nonlinear response between two sampling moments into a linear dominant part and a small nonlinear perturbation. These parts are obtained as solutions of the linear and perturbation equations respectively. Analytical integration is employed to provide an expression for the linear response. The perturbation, on the other hand, is approximated by a series of orthogonal polynomials. Combination of the two responses results in the time-efficient evaluation of weakly nonlinear behavior.

This approach has several advantages:

- Time-domain simulations simplify the modeling of systems which contain feedback via the digital domain. This is encountered, for example, in $\Delta \Sigma$ modulators. Note that a transient and not only a steady-state solution is calculated. Frequencydomain approaches, for example harmonic balance [4] or Volterra series [8, 2], are more suited for calculating steady-state solutions.

- It is straightforward to take into account effects of clock jitter since it is not assumed that the width of the time interval is fixed. To include jitter effects, sampling points are generated by a random process. Other time-domain simulation techniques (e.g. [6]) which derive an analytical expression for 
the state values between two sampling moments, for example using the Z-transform, can only model effects of clock jitter by approximated linear behavior.

- Sampled nonlinear circuits can be simulated with general time-marching methods, for example using VHDL-AMS simulators or MATLAB/Simulink. High accuracy can be achieved at the expense of very time-consuming simulations. The proposed approach reduces the simulation time while still achieving a high accuracy.

- Thermal noise can easily be added to the timedomain model. Random noise processes can be converted to multivariate stochastic variables if they are filtered in the same way as the input signals and subsequently sampled.

This paper is organized as follows. Section 2 introduces the model for characterizing weakly nonlinear systems. It is explained how this set of equations is split up into a dominant and a perturbation part. The analytical integration method to solve the first set is explained in section 3 . The approximation method for the perturbation equations is elaborated in section 4 . In section 5 two examples of the simulation method are presented. Conclusions are drawn in section 6.

\section{Characterizing weakly nonlinear systems}

A $n$-th order linear system can be modeled by a set of state-space equations which describe the behavior in terms of $n$ state variables [1]. To describe a weakly nonlinear system, these linear equations should be extended resulting in an extended state-space model.

\subsection{Extended state-space model}

Analog integrated circuits exhibiting weakly nonlinear behavior can be modeled using basic nonlinear elements like a nonlinear conductance, capacitance or transconductance which are described by a polynomial [7]. For example, a weakly nonlinear transconductance of order $N$ is characterized as follows:

$$
i(t)=g_{m} \cdot\left[v(t)+\alpha_{2} v^{2}(t)+\ldots+\alpha_{N} v^{N}(t)\right],
$$

where $\alpha_{2}, \ldots, \alpha_{N}$ are distortion coefficients. $v(t)$ is the control variable of the nonlinearity. In general, the characteristic equation is a multivariate polynomial in multiple control variables.

Figure 1 depicts an example of a nonlinear integrator and its macromodel. In this example, there are three control voltages $\left(v_{C}, v_{R}\right.$ and $\left.v_{1}\right)$ but only two
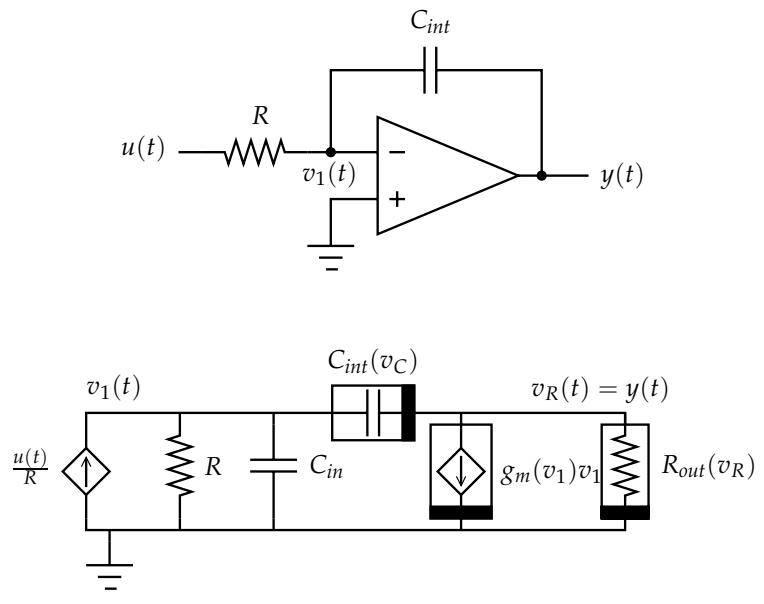

$$
\begin{aligned}
C_{\text {int }}\left(v_{C}\right) & =C_{\text {int }_{0}}\left(1+\alpha_{2} v_{C}+\cdots+\alpha_{N} v_{C}^{N-1}\right), \\
g_{m}\left(v_{1}\right) & =g_{m_{0}}\left(1+\beta_{2} v_{1}+\cdots+\beta_{N} v_{1}^{N-1}\right), \\
G_{\text {out }}\left(v_{R}\right) & =G_{\text {out }_{0}}\left(1+\gamma_{2} v_{R}+\cdots+\gamma_{N} v_{R}^{N-1}\right) .
\end{aligned}
$$

Figure 1. Example of a weakly nonlinear model for an integrator of order $N$.

state variables. In general, control voltages are linear combinations of the state and input variables which can be represented by transformation matrices:

$$
\begin{aligned}
& \boldsymbol{\theta}(t)=\mathbf{T}^{q} \cdot \boldsymbol{q}(t), \\
& \boldsymbol{\rho}(t)=\mathbf{T}^{u} \cdot \boldsymbol{u}(t),
\end{aligned}
$$

with $\boldsymbol{q}(t)$ the (linear) state variables, $\boldsymbol{u}(t)$ input signals, $\boldsymbol{\theta}(t)$ and $\boldsymbol{\rho}(t)$ control voltages and $\mathbf{T}^{q}$ and $\mathbf{T}^{u}$ transformation matrices. Note that products of control variables which are encountered in multivariate characteristic polynomials, can be converted to a sum of powers of state variables.

For the example of Figure 1, the state transformation matrix $\mathbf{T}^{q}$ is defined as follows:

$$
\boldsymbol{\theta}(t)=\left[\begin{array}{l}
v_{1}(t) \\
v_{C}(t) \\
v_{R}(t)
\end{array}\right]=\left[\begin{array}{cc}
1 & 0 \\
0 & 1 \\
1 & -1
\end{array}\right] \cdot\left[\begin{array}{l}
v_{1}(t) \\
v_{C}(t)
\end{array}\right]=\mathbf{T}^{q} \boldsymbol{q}(t),
$$

where $v_{1}(t)$ and $v_{C}(t)$ are chosen as state variables.

Based on the linear state-space equations, the mathematical description of a weakly nonlinear system with output $\boldsymbol{y}(t)$ can be written as a set of extended state-space equations:

$$
\frac{\mathrm{d} \boldsymbol{q}(t)}{\mathrm{d} t}+\sum_{k=2}^{N} \mathbf{P}_{k} \frac{\mathrm{d} \boldsymbol{\theta}^{(k)}(t)}{\mathrm{d} t}=\mathbf{A}_{1} \boldsymbol{q}(t)+\mathbf{B}_{1} \boldsymbol{u}(t)
$$




$$
\begin{aligned}
& +\sum_{k=2}^{N} \mathbf{A}_{k} \boldsymbol{\theta}^{(k)}(t)+\sum_{k=2}^{N} \mathbf{B}_{k} \boldsymbol{\rho}^{(k)}(t), \\
\boldsymbol{y}(t)=\mathbf{C}_{1} \boldsymbol{q}(t)+ & \mathbf{D}_{1} \boldsymbol{u}(t) \\
& +\sum_{k=2}^{N} \mathbf{C}_{k} \boldsymbol{\theta}^{(k)}(t)+\sum_{k=2}^{N} \mathbf{D}_{k} \boldsymbol{\rho}^{(k)}(t),
\end{aligned}
$$

where the system is characterized by the parameters of the matrices $\mathbf{P}_{k}, \mathbf{A}_{k}, \mathbf{B}_{k}, \mathbf{C}_{k}$ and $\mathbf{D}_{k} . N$ is the distortion order of the weakly nonlinear system. The superscript ${ }^{(k)}$ of a vector $v^{(k)}(t)$ indicates that each element of the vector should be raised to the power $k$.

Using Kirchoff's laws, the extended state-space model for the example in Figure 1 can be derived:

$$
\begin{aligned}
& \frac{\mathrm{d} \boldsymbol{q}(t)}{\mathrm{d} t}+\sum_{k=2}^{N}\left[\begin{array}{ccc}
0 & 0 & 0 \\
0 & \alpha_{k} & 0
\end{array}\right] \frac{\mathrm{d} \boldsymbol{\theta}^{(k)}(t)}{\mathrm{d} t}= \\
& {\left[\begin{array}{ccc}
-\frac{G+g_{m_{0}}+G_{\text {out }}}{C_{\text {in }}} & \frac{G_{\text {out }}}{C_{\text {in }}} \\
\frac{g_{m_{0}}+G_{\text {out }_{0}}}{C_{\text {int }}} & -\frac{G_{\text {out }}}{C_{\text {int }}}
\end{array}\right] \boldsymbol{q}(t)+\left[\begin{array}{c}
-\frac{G}{C_{\text {in }}} \\
0
\end{array}\right] u(t)} \\
& +\sum_{k=2}^{N}\left[\begin{array}{ccc}
-\frac{g_{m_{0}} \beta_{k}}{C_{\text {in }}} & 0 & -\frac{G_{\text {out }} \gamma_{k}}{C_{\text {in }}} \\
\frac{g_{m_{0}} \beta_{k}}{C_{\text {int }}} & 0 & \frac{G_{\text {out }} \gamma_{k}}{C_{\text {int }}}
\end{array}\right] \boldsymbol{\theta}_{n}^{(k)}(t) .
\end{aligned}
$$

All parameters are defined in Figure 1. The control variables $\boldsymbol{\theta}(t)$ are defined by state transformation (3).

\subsection{Solving the extended state-space model}

To simulate a sampled system, the response of the extended state-space model is calculated over a time interval between two sampling points provided by a sampling clock, possible with jitter. This can be simplified by taking the weakly nonlinear character into account. In this case, the nonlinear response shall be a small deviation from the linear response. So, the total response of the weakly nonlinear system can be split up into the linear response and a small perturbation:

$$
\begin{aligned}
& \boldsymbol{q}(t)=\overline{\boldsymbol{q}}(t)+\tilde{\boldsymbol{q}}(t), \\
& \boldsymbol{y}(t)=\overline{\boldsymbol{y}}(t)+\tilde{\boldsymbol{y}}(t) .
\end{aligned}
$$

When the system is part of a cascade of systems, the input signal can also be split up in a linear and a perturbation signal:

$$
\boldsymbol{u}(t)=\overline{\boldsymbol{u}}(t)+\tilde{\boldsymbol{u}}(t),
$$

where the perturbation input $\tilde{\boldsymbol{u}}(t)$ coincides with the perturbation output of the previous system.

Substituting these expressions into the extended state-space equation (4a), and simplifying, results in two sets of equations: the linear state-space equations

$$
\frac{\mathrm{d} \overline{\boldsymbol{q}}(t)}{\mathrm{d} t}=\mathbf{A}_{1} \overline{\boldsymbol{q}}(t)+\mathbf{B}_{1} \overline{\boldsymbol{u}}(t),
$$

and the perturbation equations

$$
\frac{\mathrm{d} \tilde{\boldsymbol{q}}(t)}{\mathrm{d} t}=\left[\mathbf{I}_{n}+\boldsymbol{K}(t)\right]^{-1}\left[\mathbf{A}_{1} \tilde{\boldsymbol{q}}(t)+\mathbf{B}_{1} \tilde{\boldsymbol{u}}(t)+\boldsymbol{e}(t)\right],
$$

with

$$
\begin{aligned}
\boldsymbol{e}(t) & =\sum_{k=2}^{N}\left[\mathbf{A}_{k} \boldsymbol{\theta}^{(k)}(t)+\mathbf{B}_{k} \boldsymbol{\rho}^{(k)}(t)\right]-\boldsymbol{K}(t) \frac{\mathrm{d} \overline{\boldsymbol{q}}(t)}{\mathrm{d} t}, \\
\boldsymbol{K}(t) & =\sum_{k=2}^{N} k \mathbf{P}_{k}(\operatorname{diag} \boldsymbol{\theta}(t))^{k-1} \mathbf{T}^{q},
\end{aligned}
$$

where 'diag $x^{\prime}$ converts vector $x$ to a diagonal matrix.

For each of these two sets of equations a different solution strategy is followed. Whereas for the linear set an analytical solution can be found, for the perturbation set an approximation method must be applied.

\section{Solution of the linear equations}

First, the linear state-space equations (7) are solved to determine the dominant response of the system. This can be done analytically based on the eigenstructure of the system matrix $\mathbf{A}_{1}$.

There are different formulations of the solution of the differential equation possible, for example using the exponential of $\mathbf{A}_{1}$ [1]. The formulation used in this work, however, is chosen to make it possible both to reuse a part of the calculation throughout the simulation and to employ analytical integration.

To find the response of (7) at time $t$ with initial conditions $\overline{\boldsymbol{q}}\left(t_{0}\right)$, one has to sum all contributions of all eigenvalues. One can prove that a real eigenvalue $\lambda_{k}$ of the system matrix $\mathbf{A}_{1}$ gives rise to the terms

$$
\begin{aligned}
\mathbf{Q}_{k, m}(\Delta t)^{m} & \mathrm{e}^{\lambda_{k} \Delta t} \overline{\boldsymbol{q}}\left(t_{0}\right) \\
& +\mathbf{R}_{k, m} \int_{t_{0}}^{t} \frac{(t-\tau)^{m}}{m !} \mathrm{e}^{\lambda_{k}(t-\tau)} \overline{\boldsymbol{u}}(\tau) \mathrm{d} \tau,
\end{aligned}
$$

for $m=0, \ldots, c_{k}$. The contribution of a complex eigenvalue pair $\alpha_{l} \pm \mathrm{j} \beta_{l}$ are the terms

$$
\begin{aligned}
& {\left[\mathbf{P}_{l, p}^{r} \cos \left(\beta_{l} \Delta t\right)-\mathbf{P}_{l, p}^{i} \sin \left(\beta_{l} \Delta t\right)\right](\Delta t)^{p} \mathrm{e}^{\alpha_{l} \Delta t} \overline{\boldsymbol{q}}\left(t_{0}\right)} \\
& +\mathbf{S}_{l, p}^{r} \int_{t_{0}}^{t} \frac{(t-\tau)^{m}}{m !} \mathrm{e}^{\alpha_{l}(t-\tau)} \cos \left[\beta_{l}(t-\tau)\right] \overline{\boldsymbol{u}}(\tau) \mathrm{d} \tau \\
& -\mathbf{S}_{l, p}^{i} \int_{t_{0}}^{t} \frac{(t-\tau)^{m}}{m !} \mathrm{e}^{\alpha_{l}(t-\tau)} \sin \left[\beta_{l}(t-\tau)\right] \overline{\boldsymbol{u}}(\tau) \mathrm{d} \tau,
\end{aligned}
$$


for $l=0, \ldots, d_{l}$. Here, $c_{k}+1$ and $d_{l}+1$ are the orders of the generalized eigenspace of the real and complex eigenvalues respectively. $\Delta t$ denotes the width of the time interval.

The matrices $\mathbf{Q}_{k, m}, \mathbf{R}_{k, m}, \mathbf{P}_{l, p}^{r}, \mathbf{P}_{l, p}^{i}, \mathbf{S}_{l, p}^{r}$ and $\mathbf{S}_{l, p}^{i}$ contain coefficients depending on the system. They are calculated at the beginning of the simulation. So, although it may be tedious to accurately calculate these matrices, overall simulation time is not increased accordingly since the calculation must be done only once. Substituting the expression for the general solution into the state-space equations, and equalizing orthogonal terms, shows that the columns of these coefficient matrices are elements of the null space of $\left(A_{1}-\lambda_{k} \mathbf{I}_{n}\right)^{c_{k}-m+1}$ or $\left(A_{1}-\left(\alpha_{l}+\mathrm{j} \beta_{k}\right) \mathbf{I}_{n}\right)^{d_{l}-p+1}$.

Analytical expressions are provided to calculate the integrals in the terms (9) for commonly used input signals. These analytical calculation of the integrals reduces simulation time compared to numerical evaluations. Together with the reuse of the coefficient matrices, this results in time-efficient simulations.

Furthermore, analytical integration has the advantage that input signals may be discontinuous. For example, to model initial charge of capacitors at the start of the time interval in a sampled system, Dirac impulses can be used as input signals [9].

\section{Solution of the perturbation equations}

Once the dominant behavior has been determined, the deviation of this response should be calculated by solving equations (8). The total response is then calculated as the sum of the linear and perturbation parts.

The perturbation equations can be written in the standard form of differential equations:

$$
\frac{\mathrm{d} \tilde{\boldsymbol{q}}(t)}{\mathrm{d} t}=\boldsymbol{F}(\tilde{\boldsymbol{q}}(t), t) .
$$

Furthermore, the time interval $\left[t_{0}, t_{1}\right]$ is normalized to a standard interval (in this paper $[-1,1]$ ) using the transformation

$$
\xi=\alpha t+\beta,
$$

resulting in the normalized perturbation equations in $\hat{\boldsymbol{q}}(\xi)=\tilde{\boldsymbol{q}}\left(\frac{\xi-\beta}{\alpha}\right):$

$$
\frac{\mathrm{d} \hat{\boldsymbol{q}}(\xi)}{\mathrm{d} \xi}=\frac{1}{\alpha} \boldsymbol{F}\left(\hat{\boldsymbol{q}}(\xi), \frac{\xi-\beta}{\alpha}\right) .
$$

Generally, an analytical solution cannot be found for these equations. Instead, the signals can be approximated over the entire time interval:

$$
\hat{\boldsymbol{q}}(\xi)=\sum_{i=0}^{M} \hat{Q}_{i} p_{i}(\xi)
$$

where the signals $\left\{p_{0}(\xi), \ldots, p_{M}(\xi)\right\}$ are the base signals of the approximation. In this work, orthogonal polynomials are chosen as base functions. This set has several advantages compared to other sets:

- Using polynomials simplifies function evaluations compared to other types of base functions.

- One could also use Taylor series as polynomial approximation. The advantage of series of orthogonal polynomials is that they provide an approximation over the entire time interval. Taylor series, on the other hand, approximate the signal only at the beginning of the interval. The main point of interest, however, in the case of sampled systems is the end point of the interval.

In this work, Chebyshev polynomials are used as base functions.

To find the coefficients $\hat{Q}_{i}$ of the polynomial series (13), one could substitute the approximation into the differential equations and equalize corresponding terms. This method has some disadvantages:

- Nonlinear behavior requires the calculation of powers of the approximated signal in terms of base functions of order 0 to $M$. Such expressions are nonlinear and complicated. Furthermore, they are approximations which introduce an error on the differential which is not correct anywhere in the interval. Especially in the end point of the interval, a large error can be the result.

- All input signals, including the linear approximation, should be approximated by series of the base functions.

Another approach consists in writing down the differential equations in $M$ collocation points. Together with the initial conditions, a nonlinear system is obtained of as many equations as with the method described above. Since this is not easier or more difficult to solve and it does not suffer of the aforementioned disadvantages, this method is our first choice to calculate the coefficients of the approximation.

There are $M$ collocation points to be chosen within the standard interval. For Chebyshev polynomials, it is advantageous to select the points

$$
\xi_{j}=\cos \left(\frac{(j-1) \pi}{M-1}\right), \quad j=1, \ldots, M,
$$

if $M \neq 1$. For $M=1$, the end point is selected as collocation point (which is 1 in the standard interval).

Now the differential equations are written down in each point $\xi_{j}$. The left-hand side of (12) becomes

$$
\frac{\mathrm{d} \hat{\boldsymbol{q}}(\xi)}{\mathrm{d} \xi}=\sum_{i=0}^{M} \hat{\boldsymbol{Q}}_{i} \frac{\mathrm{d} p_{i}\left(\xi_{j}\right)}{\mathrm{d} \xi}
$$


with $\frac{\mathrm{d} p_{i}\left(\xi_{j}\right)}{\mathrm{d} \xi^{\tau}}$ the derivative of the base function of order $i$ in $\xi_{j}$. For Chebyshev polynomials and the choice of collocation points of (14), one can prove that

$$
\frac{\mathrm{d} p_{i}\left(\xi_{j}\right)}{\mathrm{d} \xi}= \begin{cases}i+2 i \sum_{l=1}^{\frac{i-1}{2}} \cos \left(\frac{2 l(j-1) \pi}{M-1}\right) & i \text { odd }, \\ 2 i \sum_{l=1}^{\frac{i}{2}} \cos \left(\frac{(2 l-1)(j-1) \pi}{M-1}\right) & i \text { even. }\end{cases}
$$

These values should only be calculated at the beginning of the simulation, since the interval is always normalized to the same standard interval.

For the right-hand side of (12) the values of the normalized perturbation signals $\hat{\boldsymbol{q}}(\xi)$ should be written as a function of the coefficients $\hat{Q}_{i}$. All coefficients can be lumped together within the coefficient vector $\hat{Q}$ :

$$
\hat{\boldsymbol{Q}}=\left[\begin{array}{llll}
\hat{\boldsymbol{Q}}_{0}^{T} & \hat{\boldsymbol{Q}}_{1}^{T} & \cdots & \hat{\boldsymbol{Q}}_{M}^{T}
\end{array}\right]^{T} .
$$

The values of $\hat{\boldsymbol{q}}(\xi)$ in the collocation point $\xi_{j}$ can be written as a matrix multiplication:

$$
\hat{\boldsymbol{q}}\left(\xi_{j}\right)=\mathcal{P}\left(\xi_{j}\right) \cdot \hat{Q},
$$

with

$$
\mathcal{P}(\xi)=\left[\begin{array}{lll}
\operatorname{diag}_{n} p_{0}(\xi) & \cdots & \operatorname{diag}_{n} p_{M}(\xi)
\end{array}\right] .
$$

The operator ' $\operatorname{diag}_{n}$ ' is defined as the Kronecker product between the identity matrix and a scalar, vector or matrix (i.e. $\operatorname{diag}_{n} \phi=\mathbf{I}_{n} \otimes \phi$ ). The function values of a Chebyshev polynomial in the points of (14) are

$$
p_{i}\left(\xi_{j}\right)=\cos \left(\frac{i(j-1) \pi}{M-1}\right) .
$$

Using these expressions, the set of differential equations written down in the collocation points together with the initial conditions can be converted to a system of (nonlinear) algebraic equations:

$$
\begin{aligned}
& \boldsymbol{V}(\hat{\boldsymbol{Q}})= \\
& {\left[\begin{array}{c}
\mathcal{P}^{\prime}\left(\xi_{1}\right) \\
\vdots \\
\mathcal{P}^{\prime}\left(\xi_{M}\right) \\
\mathcal{P}(-1)
\end{array}\right] \hat{\boldsymbol{Q}}-\frac{1}{\alpha}\left[\begin{array}{c}
\boldsymbol{F}\left(\mathcal{P}\left(\xi_{1}\right), \frac{\xi_{1}-\beta}{\alpha}\right) \\
\vdots \\
\boldsymbol{F}\left(\boldsymbol{\mathcal { P }}\left(\xi_{M}\right), \frac{\xi_{M}-\beta}{\alpha}\right) \\
\mathbf{0}
\end{array}\right]=\mathbf{0},}
\end{aligned}
$$

with $\mathcal{P}^{\prime}(\xi)$ defined similar to $\mathcal{P}(\xi)$ but with derivatives instead of function values of the base functions.

An approximation of the perturbation coefficients can be found by solving the set of nonlinear algebraic equations using Newton-Rhaphson iterations. Since the system is weakly nonlinear, it should have an approximately linear behavior. So, a first-order approximation is

$$
\hat{Q} \approx-\left[\frac{\mathrm{d} \boldsymbol{V}(\mathbf{0})}{\mathrm{d} \hat{Q}}\right]^{-1} \cdot \boldsymbol{V}(\mathbf{0}),
$$

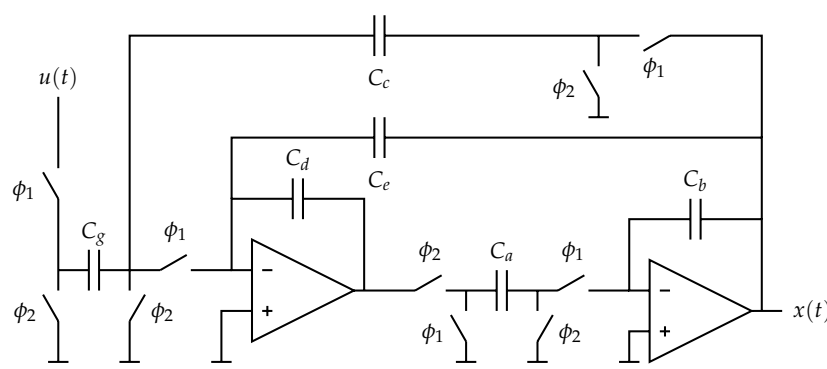

Figure 2. Fleischer-Laker switched-capacitor biquad used in the first experiment [5].

with $\frac{d \boldsymbol{V}(\mathbf{0})}{\mathrm{d} \hat{\boldsymbol{Q}}}$ the jacobian of the vector $\boldsymbol{V}(\hat{\boldsymbol{Q}})$. Although more accuracy can be obtained by performing multiple Newton iterations, (21) is a good approximation for weakly nonlinear circuits. No significant improvement was found when two or three iterations were performed in the experiments described in section 5 . Note that this means that the coefficients are the result of the solution of one set of linear equations.

The complexity of this algorithm depends on how efficiently the jacobian in (21) can be calculated. It is straightforward to calculate $\frac{\mathrm{d} V(\mathbf{0})}{\mathrm{d} \hat{Q}}$ once the jacobian of $\boldsymbol{F}(\hat{\boldsymbol{q}})$ of (12) is evaluated in all collocation points. For this evaluation, one can determine an expression similar to (8), i.e. the calculation of the jacobians is simplified by storing terms common to the jacobian and the function values, especially the factor $\left[\mathbf{I}_{n}+\boldsymbol{K}(\hat{\boldsymbol{q}})\right]^{-1}$. Furthermore, parts of both functions should only be calculated once at the beginning of the simulation.

\section{Experimental results}

An implementation of the above algorithm has been developed to verify its accuracy and simulation time. The model has a discrete-time computational model which makes it straightforward to implement it as a SystemC module.

First, the switched-capacitor circuit depicted in Figure 2 is analyzed. The circuit is clocked at a sampling rate of $128 \mathrm{MHz}$. It is assumed that capacitors $C_{b}$ and $C_{d}$ are modeled by third-order characteristic polynomials. Figure 3 shows the signal-to-noise-anddistortion ratio $(S N D R)$ at $y(t)$ when the input is a sine wave of about $1 \mathrm{MHz}$. The numbers between parentheses are the normalized simulation times. The timemarching algorithm used for comparison is a standard Runge-Kutta algorithm. Such an algorithm is used by general simulation approaches like MATLAB simula- 


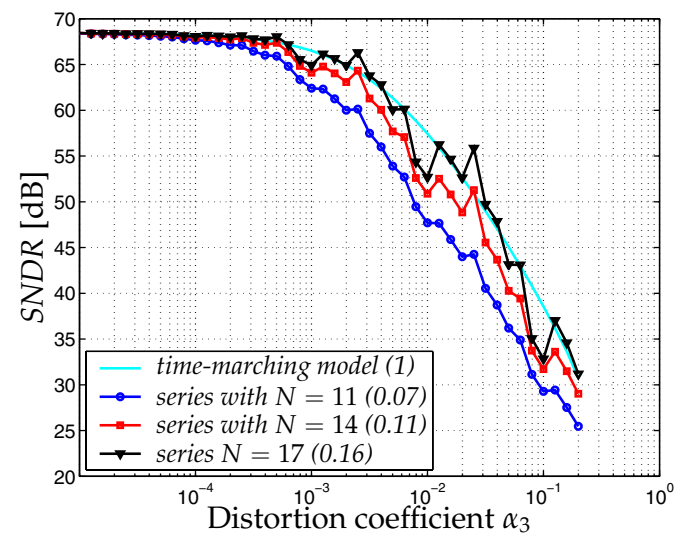

Figure 3. Effect of nonlinear capacitors $C_{b}$ and $C_{d}$ in the circuit of Figure 2.

tions ${ }^{1}$. One can conclude that, although high orders of the polynomial series are required for good approximation, simulations are about six times faster.

As second experiment, the model is used to calculate the SNDR degradation in a third-order singleloop $\Delta \Sigma \mathrm{A} / \mathrm{D}$-converter with an oversampling ratio of 128. At each time step, the SystemC module calculates the new states of the loop filter. These values are the inputs of a quantizer module which produces a bit stream. Feedback is provided by a DAC by generation of pulses at sampling moments. The integrator model is shown in Figure 1. Figure 4 depicts the drop in SNDR as a function of the distortion coefficient of the integrating capacitor. Comparison is made with a time-marching simulation of a VHDL-AMS model ${ }^{2}$. Due to the feedback structure of a $\Delta \Sigma$ modulator and its tolerance to circuit non-idealities, lower-order approximations already result in a good accuracy. Also for this experiment, a speed-up factor of about six is achieved.

\section{Conclusions}

Time-efficient simulations of sampled systems are a great aid in the systematic analysis and synthesis of switched subsystems frequently used in analog and mixed-signal integrated circuits.

The simulation algorithm described in this paper is applicable for sampled weakly nonlinear circuits and achieves a reduction of the simulation time by two means. First, the dominant behavior is calculated analytically. Then, the deviation of the linear response is

\footnotetext{
${ }^{1}$ The Runge-Kutta algorithm is included in the SystemC module to make a fair comparison.

${ }^{2}$ The simulator used is AdvanceMS of Mentor Graphics ${ }^{\circledR}$.
}

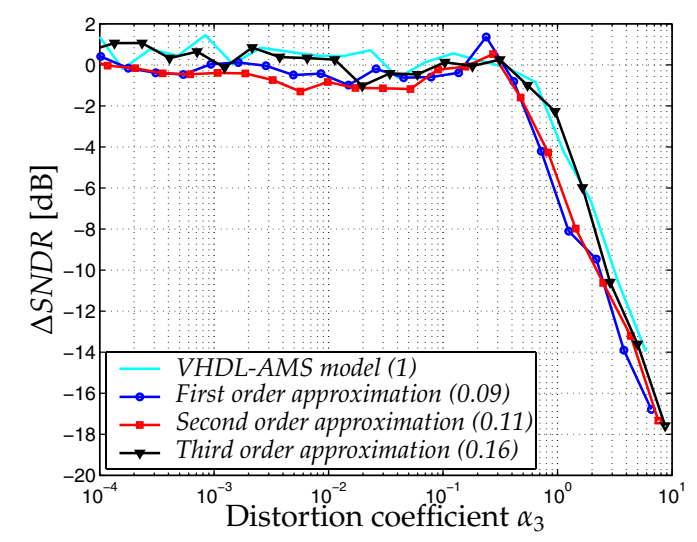

Figure 4. $S N D R$ degradation due to nonlinear distortion in $\Delta \Sigma$ modulator.

calculated as a small perturbation. Since the perturbation should be small, it can be approximated by a finite polynomial series whose coefficients are calculated by solving a set of linear equations. Experimental results of a switched-capacitor filter and a continuous-time $\Delta \Sigma$ converter have shown that the proposed method is more time-efficient than time-marching algorithms used by, for example, MATLAB and VHDL-AMS simulators, while giving similar results.

\section{References}

[1] W.-K. Chen, editor. The Circuits and Filters Handbook. CRC Press, 1995.

[2] P. Dobrovolný et al. "Analysis and Compact Behavioral Modeling of Nonlinear Distortion in Analog Communication Circuits". IEEE Trans. CAD of Integrated Circuits $\mathcal{E}$ Systems, 22(9):1215-1227, Sept. 2003.

[3] G. G. Gielen and R. A. Rutenbar. "Computer-Aided Design of Analog and Mixed-Signal Integrated Circuits". Proc. of the IEEE, 88(12):1825-1849, Dec. 2000.

[4] K. S. Kundert and A. Sangiovanni-Vincentelli. "Simulation of Nonlinear Circuits in the Frequency Domain". IEEE Trans. CAD, 5(4):521-535, Oct. 1986.

[5] K. Laker and W. Sansen. Design of Analog Integrated Circuits and Systems. McGraw-Hill, Inc., 1994.

[6] P. Malcovati et al. "Behavorial Modeling of SwitchedCapacitor Sigma-Delta Modulators". IEEE Trans. Circuits $\mathcal{E}$ Systems-I: Fundamental Theory $\mathcal{E}$ Applications, 50(3):352-364, Mar. 2003.

[7] P. Wambacq and W. Sansen. Distortion Analysis of Analog Integrated Circuits. Kluwer Academic Publishers, 1998.

[8] F. Yuan and A. Opal. "Distortion Analysis of Periodically Switched Nonlinear Circuits Using Time-Varying Volterra Series". IEEE Trans. Circuits E Systems-I: Fundamental Theory $\mathcal{E}$ Applications, 48(6):726-738, June 2001.

[9] F. Yuan and A. Opal. "Computer Methods for Switched Circuits". IEEE Trans. Circuits \& Systems-I: Fundamental Theory E Applications, 50(8):1013-1024, Aug. 2003. 